\title{
Maintenance of a positive outlook during acute stress protects against pro-inflammatory reactivity and future depressive symptoms
}

\author{
K. Aschbacher ${ }^{1}$, E. Epel ${ }^{1}$, O.M. Wolkowitz ${ }^{1}$, A.A. Prather ${ }^{1}$, E. Puterman ${ }^{1}$, and F.S. \\ Dhabhar $2,3,4$ \\ ${ }^{1}$ Department of Psychiatry, University of California San Francisco, CA \\ ${ }^{2}$ Department of Psychiatry and Behavioral Sciences, Stanford University, CA \\ ${ }^{3}$ Stanford Institute for Immunity, Transplantation and Infection, Stanford University, CA \\ ${ }^{4}$ Stanford Cancer Center, Stanford University, Stanford, CA
}

\section{Abstract}

Cognitive and affective responses to acute stress influence pro-inflammatory cytokine reactivity, and peripheral cytokines (particularly lnterleukin-1 beta (IL-1 $\beta$ )), can act on the brain to promote depressive symptoms. It is unknown whether acute stress-induced changes in positive affect and cognitions (POS) and pro-inflammatory reactivity predict future depressive symptoms. We examined acute stress responses among women, to determine prospective predictors of depressive symptoms. Hypotheses: 1) Stress-induced decreases in POS will be associated with stress-related increases in circulating IL-1 $\beta$. 2) Acute stress-induced decreases in POS and increases in IL-1 $\beta$ reactivity will predict increases in depressive symptoms one year later. Thirty-five postmenopausal women were exposed to acute stress with the Trier Social Stress Task (TSST) and provided blood samples under resting conditions and 30 minutes after the conclusion of the TSST, which were assayed for IL- $1 \beta$. IL- $1 \beta$ reactivity was quantified as post minus pre-TSST. Failure to maintain POS was quantified as the decrease in POS during the TSST. Change in depressive symptoms from the study baseline to the following year was determined. Greater acute stressinduced declines in POS were significantly associated with increased IL-1 $\beta$ reactivity $(p \leq 02)$, which significantly predicted increases in depressive symptoms over the following year $(p<.01)$, controlling for age, body mass index, chronic stress, antidepressant use and baseline depressive symptoms. IL- $1 \beta$ reactivity was a significant mediator of the relationship between POS decline and future increases in depressive symptoms $(p=.04)$. Difficulty maintaining positivity under stress

(c) 2011 Elsevier Inc. All rights reserved.

Corresponding Authors: Kirstin Aschbacher, Ph.D., Postdoctoral Fellow, University of California San Francisco, Department of Psychiatry, School of Medicine, 3333 California Street, Suite 465, San Francisco, CA 94143-0848, Tel: 415-502-7908, Fax: 415-476-7744, kirstin.aschbacher@ucsf.edu. Firdaus S. Dhabhar, Ph.D., Associate Professor, Stanford University, Department of Psychiatry \& Behavioral Sciences, Institute for Immunity, Transplantation \& Infection, Stanford University School of Medicine, 259 Campus Drive, MC 5135, Stanford, CA 94305-5135, Tel/Fax: 650-736-8565, dhabhar@gmail.com.

Publisher's Disclaimer: This is a PDF file of an unedited manuscript that has been accepted for publication. As a service to our customers we are providing this early version of the manuscript. The manuscript will undergo copyediting, typesetting, and review of the resulting proof before it is published in its final citable form. Please note that during the production process errors may be discovered which could affect the content, and all legal disclaimers that apply to the journal pertain. 
and heightened pro-inflammatory reactivity may be markers and/or mechanisms of risk for future increases in depressive symptoms.

\section{Keywords}

cytokine; pro-inflammatory; interleukin-1 beta; acute stress; reactivity; positive affect; depressive symptoms

\section{Introduction}

Depressive disorders are highly prevalent and place a substantial burden on society. They nearly double the risk of premature mortality (Barth et al., 2004; Cuijpers and Smit, 2002), contribute to run-away health care budgets (Unutzer et al., 2009), and cost employers billions of dollars each year in lost productivity (Stewart et al., 2003). Moreover, the increased morbidity and economic costs are nearly as great for subclinical levels of depressive symptoms (Cuijpers et al., 2007; Cuijpers and Smit, 2002). Despite the prevalence and importance of treating depressive symptomology, one-third of patients with Major Depressive Disorder (MDD) fail to experience symptom remission following multiple courses of antidepressant treatment (Rush et al., 2006). This suggests a need to better elucidate both the psychological and biological mechanisms underlying depressive symptoms, in order to develop novel approaches to treatment.

The "Cytokine Hypothesis of Depression" proposes that inflammation may be a causal mechanism in the development of depressive symptomology (Dantzer et al., 2008; DellaGioia and Hannestad, 2010; Miller et al., 2009). Pro-inflammatory cytokines, such as Interleukin (IL)-1 $\beta$, IL-6 and Tumor Necrosis Factor alpha (TNF- $\alpha$ ), are known to induce sickness behavior, a set of behavior changes that overlap with depressive symptomology (e.g., fatigue, social withdrawal, loss of interest) (Dantzer et al., 2008). A number of studies have found that patients with depressive disorders have a heightened pro-inflammatory milieu relative to controls (Dhabhar et al., 2009; Maes et al., 1997; Thomas et al., 2005). These elevations persist after antidepressant treatment among non-responders (Maes et al., 1997), and correlate with symptom severity and episode duration in older depressed adults (Thomas et al., 2005). Endotoxin administration in humans provokes in vivo cytokine reactivity, which provokes increases in depressive symptoms over the following hours to days (DellaGioia and Hannestad, 2010). Moreover, in vitro LPS-induced production of IL-1 family cytokines was prospectively associated with 5-year increases in depressive symptoms among older adults (van den Biggelaar et al., 2007). While the experimental endotoxin model has provided rich insights, its translational potential is limited because it explains the occurrence of depression due to a general medical disorder better than depression among healthy individuals.

Acute cytokine reactivity to laboratory stress tasks may provide a valuable model for understanding the mechanisms and individual predictors of stress-related depression. Stressful life events frequently precede depressive episodes (Kendler et al., 1998), and stressors are also known to provoke pro-inflammatory cytokine release (Altemus et al., 2001; Steptoe et al., 2007). However, relatively few studies have examined acute stress- 
induced cytokine reactivity in relation to depressive disorders or symptoms (Miller et al., 2005; Pace et al., 2006). None of these studies investigated prospective associations, to determine whether pro-inflammatory reactivity might be a marker of long-term vulnerability. Hence, this study investigates whether cytokine reactivity to an acute laboratory stress task predicts subsequent increases in depressive symptoms over one year.

In a meta-analysis of acute stress effects on cytokine reactivity, IL- $1 \beta$ exhibited the largest effect size ( $r=0.58$ ), followed by IL-6 ( $r=.19)$, while TNF- $a$ increases in response to stress were not significant (Steptoe et al., 2007). Animal models further underscore the central role of IL-1 $\beta$ in stress-induced depression (Goshen et al., 2008; Nguyen et al., 1998). Notably, many of the depressive behavioral effects of LPS can be blocked by pretreatment with IL-1 receptor antagonist (IL-1ra) (Bluthe et al., 1992). This study focused on IL-1 $\beta$ as a key candidate cytokine that may help explain why, and for whom, stress is likely to promote depressive symptoms. Secondarily, the study also examined IL-6, which may also play an important role in depressive disorders (Dhabhar et al., 2009; Pace et al., 2006) and is regulated by IL-1 $\beta$ (Dinarello, 2009).

The magnitude of physiological stress responses reflects, in part, a trait-like individual characteristic (Cohen and Hamrick, 2003), with the capacity to predict elevations in biomarkers of disease processes (Brydon and Steptoe, 2005; Matthews et al., 2004). Nonetheless, the way individuals appraise their ability to manage stress also impacts their affective, cognitive and biological stress responses (Tomaka et al., 1997). Increases in anxiety and anger during an acute laboratory stress task have been associated with greater concomitant pro-inflammatory reactivity (Carroll et al., 2011). In contrast, little is known about whether the ability to maintain positively oriented cognitions and affect (e.g., confidence, hope or challenge) during an acute stressor is associated with decreased proinflammatory reactivity or susceptibility to depressive symptoms. Appraising stressors as challenges rather than threats may buffer stress reactivity and promote salutary physiological processes (Epel et al., 1998).

The current study investigates a PsychoNeurolmmunological model of how the loss of a positive outlook during stress may regulate inflammatory reactivity and predict subsequent mood. This inquiry focuses on two hypotheses: 1) Decreasing positivity in response to an acute laboratory stress task will be significantly related to greater pro-inflammatory IL-1 $\beta$ reactivity during the stressor, and 2) Greater stress-induced declines in positivity and increases in pro-inflammatory IL- $1 \beta$ reactivity will be significantly associated with greater susceptibility to depressive symptom increases over the following year.

\section{Methods}

\section{Participants}

All participants provided written informed consent, and the study protocol was approved by the Institutional Review Board of the University of California San Francisco. The sample was drawn from a larger cohort of post-menopausal women who participated in a prospective study of stress and immunological aging. Forty-eight women took part in an acute laboratory stress task, completed psychological ratings and provided blood samples for 
assays of pro-inflammatory cytokine reactivity. Three participants could not be included because they declined to continue or developed a headache. The study criteria excluded participants with chronic medical conditions - including cardiovascular disease, cancer, and diabetes - as well as participants who were current smokers or used medications known to affect stress hormones (e.g., corticosteroid or gonadal steroid-containing). Antidepressant use was not an exclusion criterion, but was a covariate. To confirm health status at baseline, participants were screened by self-report and also passed a physical exam by a physician and a panel of standard laboratory tests (i.e., normal glucose, electrolytes, and function of the thyroid, liver and kidneys). This eligibility visit blood draw was conducted in the morning, fasting, and the blood was assessed for cytokine levels. One week later, participants returned for an afternoon stress test session (see below).

\section{The Acute Stress Test Protocol}

Participants ate a standardized lunch, had an antecubital intravenous catheter inserted around $1 \mathrm{pm}$, and then rested for one hour while listening to relaxing music on headphones. The Trier Social Stress Task (TSST) was conducted (Kirschbaum et al., 1993), while blood samples were collected to determine pro-inflammatory reactivity. The TSST included both a speech (about strengths and weaknesses rather than a job interview due to the fact that some older individuals were retirees) and a math task (subtraction of consecutive prime numbers). The stress task lasted 20 minutes total (5-minutes each: introduction to tasks, preparation for speech task, giving speech, math task), and was conducted in the presence of two stonyfaced evaluators. Blood draws for cytokine levels were conducted at 0 and 50 minutes following stressor onset.

\section{Maintenance of a Positive Outlook}

Affective and cognitive indicators that reflect the maintenance of a positive outlook during acute stress (POS) were assessed before (pre) and immediately after (post) the TSST (i.e., representing a 20-minute time-span). The construct of POS was assessed with an 8-item questionnaire including two sub-domains: affect and cognition. The positive affect subdomain consisted of 4 items that asked participants how hopeful, confident, eager, and excited they felt prior to the stress task. These items were rated on a 5-point Likert scale ranging from $0=$ "not at all" to $4=$ "a great deal." The cognitive positive arousal sub-domain consisted of 4 items (9-point Likert agreement scale), which tapped into confidence in one's ability to cope well with the task: e.g., "I will be able to complete the upcoming tasks successfully." Items assessing post-task responses were worded similarly, but changed the verb tense to past: e.g., "I was able to complete the tasks successfully." This scale utilizes items derived from previously published studies assessing emotional and cognitive appraisals of demands and resources in response to acute stress tasks (Epel et al., 2009; Mendes et al., 2001). The total positivity score was quantified as the sum of the normalized scores for the two sub-domains, and demonstrated good internal consistency (Cronbach's alpha in the current sample $=.87$, total score). Total POS scores were quantified as the difference: post minus pre, such that higher numbers indicate increases or lesser decreases. As a validity check to verify that the POS measure was assessing positive affect, it was compared with measures from the Positive and Negative Affect Schedule (PANAS), in which participants rated how much they experienced various emotions in the past week 
(Watson et al., 1988). The positive scale includes feelings such as enthusiastic, interested, and happy, while the negative scale encompasses aversive feelings such as scared, troubled, sad, guilty and irritable. Data on the PANAS were missing for one participant.

\section{Inventory of Depressive Symptoms (IDS)}

The self-administered, 30-item IDS (Rush et al., 1986; Rush et al., 1996) was used to assess the severity of depressive symptoms at baseline and one year following. The IDS is validated for use with both clinically depressed and non-depressed individuals. It exhibits strong reliability (Cronbach's alpha $=.94)$, correlates highly with other well-established measures of depression (e.g., Hamilton Depression Rating Scale) and provides a sensitive measure of symptom severity (Rush et al., 1996). The magnitude of the increase in depressive symptoms (a primary outcome measure) was calculated as year 1 score minus baseline score.

\section{Assessment of General Health \& Health Behaviors}

Participant health was assessed by self-reported physician-diagnosed illness at study entry on the following variables potentially related to immune function: hypertension $(n=11)$, hypercholesterolemia $(n=14)$, chronic pain $(n=6)$, arthritis/osteoarthritis $(n=8)$, osteoporosis $(n=4)$, allergies $(n=17)$. No participants had autoimmune disorders. Use of the following classes of medications was also recorded and investigated for potential covariates: NonSteroidal Anti-Inflammatory Drugs (NSAIDs) $(n=8)$, antidepressants (selective serotonin reuptake inhibitors, tricyclics, or atypical $(n=6))$, anti-hypertensives $(n=13)$, statins $(n=8)$, thyroid medications $(n=3)$, and anti-oxidant supplements $(n=15)$. Current smoking was an exclusion criterion, so 'former smoker' was defined as having smoked 100 or more cigarettes in one's lifetime $(n=18)$. Body Mass Index (BMI), alcohol use in the previous month (yes/no) and moderate physical activity (self-reported frequency*average duration of exercise in the previous month) were assessed.

\section{Pro-Inflammatory Activity: Interleukin-1 $\beta$ \& Interleukin-6}

Whole blood was collected into 10ml SST tubes (Becton Dickinson, Franklin Lakes, and $\mathrm{NJ}$ ). Blood was allowed to clot for 30 minutes at room temperature, and then centrifuged at $1300 \mathrm{rpm}$ for 15 minutes. Serum was collected, frozen, and stored at $-75^{\circ} \mathrm{C}$ for subsequent cytokine quantification. A high sensitivity sandwich immunoassay was used to quantify IL-1 $\beta$ and IL-6 (Meso Scale Discovery (MSD), Gaithersburg, MD). The assay sensitivity is $0.07 \mathrm{pg} / \mathrm{ml}$ and the average intra- and inter-assay coefficients of variation are $3 \%$ and $4 \%$ respectively. Although the current study hypotheses focused on IL- $1 \beta$ for the reasons previously outlined, IL-6 was included in order to investigate whether effects were specific to IL-1 $\beta$, or more generally attributable to pro-inflammatory factors.

\section{Data Analyses}

Pro-inflammatory reactivity was quantified as the change from 0 to 50 minutes, where larger scores indicate greater increases in response to acute stress. This time-period was selected as the focus because it roughly approximates the time required for increased biosynthesis through enlargement of the circulating cell pool or gene transcription-mediated processes 
(Steptoe et al., 2007). IL-1 $\beta$ and IL-6 values were log-transformed to improve the normality of the distributions. Two outliers at 0 minutes and one at 50 minutes were removed, because they were greater than four standard deviations above the mean after log-transformation. For IL-1 $\beta$, several values that were above .05 and below $0.07 \mathrm{pg} / \mathrm{ml}$ were imputed as being .06 , which maintained their rank order in the distribution. Values below .05 were coded as below the limit of detection, and separate analyses examined whether these individuals differed on key factors (see results). The resulting variables for pro-inflammatory reactivity, positive outlook and depressive symptom variables did not significantly differ from a normal distribution per the Kolmogorov-Smirnov (K-S) test.

The final sample consisted of thirty-five individuals with complete data on proinflammatory reactivity and psychological responses to acute stress. Of these, four individuals were unavailable at one-year follow-up leaving a sample size of thirty-one for the prospective analyses predicting future depressive symptoms. The primary hypotheses were tested using linear regression in which the primary covariates (age, BMI, caregivers status $(0=\mathrm{CG}, 1=\mathrm{NC})$, and antidepressant use) were entered into block 1 (one at a time, in separate regression analyses to conserve power), and the independent variable of interest was included in block 2 . Two-tailed tests with a critical alpha of .05 were used.

\section{Results}

\section{Sample Characteristics}

In the final sample of 35 participants with psychological and pro-inflammatory reactivity data, the mean age of the participants was 61 years (range: 51-75), 86\% were Caucasian, $66 \%$ had a Bachelor's degree or higher, and the median income was approximately $\$ 70,000$ / year. As a goal of the larger study was to examine chronic stress, 16 of the 35 women were providing care for a family member with dementia, while the remaining 19 were not (used as a covariate). The average BMI in the study was $26(S D=5.37)$. The mean depression score was $13.26(S D=8.73)$ on the IDS at the baseline visit and $11.79(S D=7.21)$ at the 1-year follow-up visit. Validated IDS cut-offs designed to detect significant functional impairment associated with subthreshold depression were used to categorize participants' symptom severity as mild (score 14-25) or moderate (score 26-38) (no patients had scores above moderate) for participants with with $\triangle$ IDS data (Karsten et al., 2010). The percentage of participants who met criteria were as follows: at baseline - 32\% mild, 3\% moderate, and at 12-month follow-up - 26\% mild, 3\% moderate. Absolute cytokine values at TSST baseline and 50 minutes later are given in Table 1.

\section{Determination of Covariates}

Age, BMI, caregiver status (caregiver or non-caregiver), and antidepressant use at baseline ( $1=$ yes, $0=$ no), and change in antidepressant (AD) use from baseline to 12 months $(-1=$ stopped taking, $0=$ no change, $1=$ started taking) were included as covariates in all analyses and entered sequentially to avoid over-fitting. In addition, four classes of factors were investigated for potential inclusion as covariates: 1) demographics, 2) medical conditions, 3) health behaviors and 4) medication use. Pearson correlations (for continuous factors) and t-tests (for dichotomous predictors) were used to establish whether any of these 
factors were significantly associated with the primary outcome (depressive symptoms increase $=\Delta \mathrm{IDS}$ ) or the proposed mediator (i.e., IL-1 $\beta$ reactivity).

Higher BMI at baseline was associated with a nearly significant association with increasing depressive symptoms, indicated by $\Delta \operatorname{IDS}(r=.352, p=.052)$. None of the demographic factors (i.e., age, being Caucasian, having a Bachelor's degree or better, and being at median income or above) or the health behaviors assessed significantly predicted $\Delta$ IDS or IL-1 $\beta$ reactivity in independent t-tests. No other associations between preexisting medical illnesses or medication use and either IL- $1 \beta$ reactivity or $\Delta$ IDS were found. In analyses without covariates, caregiver status was initially associated with significant decreases in depressive symptoms ( $p=.020)$; however, when controlling for basal depressive symptoms levels in a linear regression, caregiver status was no longer significantly associated with $\Delta \mathrm{IDS}(p>.20)$. The significant effect of basal depressive symptoms indicates that, on average, individuals who had higher levels of depressive symptoms at baseline decreased a few points over the following year $(\beta=-.414, t(28)=-2.329, p=.027)$, possibly due to regression to the mean or natural symptom regression.

\section{Limit of Detection Analyses}

To ensure that omitting individuals with IL-1 $\beta$ levels below the limit of detection did not unduly bias these results, we conducted analyses to determine whether individuals whose values were deemed too far below the limit to impute $(<.05 \mathrm{pg} / \mathrm{ml})^{*}$ differed from individuals with values above the limit on the major predictors, covariates and outcomes. Dichotomous variables ( 1 =below the limit of detection, $0=$ above) were created for each time-point and $t$-tests were conducted. Lower BMI was associated with a greater likelihood of being below the limit of IL-1 $\beta$ detection at baseline ( $p=.04)$. POS, basal IDS, $\Delta$ IDS, age, caregiver status, and antidepressant use did not significantly differ as a function of being below versus above the limit of detection (all $p$ 's >.05).

\section{Hypothesis $1(\mathrm{H} 1)$ : The Role of Maintaining a Positive Outlook}

A primary goal of the study is to examine the extent to which difficulty maintaining positive affect and cognitions (POS) during acute stress are associated with concurrent proinflammatory reactivity and future depressive symptoms. As a preliminary validation check, Pearson correlations were conducted to confirm that higher reported levels of POS were significantly associated with higher positive affect scores on the PANAS (respectively: prestress, $\mathrm{r}=.484, p=.004$; post-stress, $\mathrm{r}=.349, p=.043$ ), and were not significantly associated with the negative affect subscale of the PANAS (all $p$ 's $>.20$ ). The change in POS from pre to post was not significantly related to either positive or negative affect on the PANAS, suggesting that overall POS levels may capture generalized positive affect, while POS change reflects a more dynamic measure of psychological stress appraisals.

Decreasing POS during acute stress was significantly associated with higher proinflammatory IL- $1 \beta$ reactivity $\left(\beta=-.400, t(33)=-2.510, p=.017\right.$; Figure $\left.1^{\dagger}\right)$. This association

\footnotetext{
*For IL-1 $\beta$ values at fasting baseline, immediately prior to the stress task and after 50-minutes, the number of points below the limit was 4,3 , and 0 respectively.

${ }^{\dagger} \mathrm{POS}$ is a continuous variable in all analyses reported. It is dichotomized in Figure 1 to better visualize the data.
} 
remained significant when controlling for age, BMI, caregiver status, AD use, and fasting IL-1 $\beta$ baseline. Furthermore, as we hypothesized that pro-inflammatory activity may be an important biological mechanism mediating relationships between POS and depressive risk, relations between POS and $\triangle$ IDS were examined. There was a significant effect, such that decreasing POS was associated with greater $\Delta \mathrm{IDS}(\beta=-.355, t(29)=-2.047, p=.050)$, consistent with the notion that poor maintenance of POS may place individuals at risk for future increases in depressive symptoms. This association remained borderline significant $(p \leq 08)$ when controlling for all covariates sequentially as well as basal IDS $\ddagger$. No evidence of moderating effect of being a caregiver was found.

\section{H1 Follow-Up Analyses: Affective versus Cognitive Components of Positive Arousal}

As a follow-up analysis, we investigated whether the affective or cognitive subscales of POS provided a stronger or more specific determinant of pro-inflammatory reactivity and future depressive symptoms. Greater acute-stress induced declines in positive affect were significantly associated with increases in pro-inflammatory reactivity $(\beta=-.403, t(33)=$ $-2.526, p=.017$ ), and a trend toward increases in depressive symptoms, per $\Delta \mathrm{IDS}$ ( $\beta=-.313$, $t(29)=-1.772, p=.087)$. The decrease in positive cognitive assessments of one's ability to handle the speech stress task was associated with borderline significant effects on IL-1 $\beta$ reactivity $(\beta=-.298, t(33)=-1.791, p=.082)$ and no significant effects on $\Delta \mathrm{IDS}(\beta=-.288$, $t(29)=-1.623, p=.116)$. In sum, the effect sizes suggest that decreases in positive affect were a stronger predictor of both pro-inflammatory reactivity to stress and future depressive symptom risk than changes in positive cognitive appraisals of one's ability to perform well in the stress task.

\section{Hypothesis 2: IL-1 $\beta$ Reactivity to Acute Stress and 1-Year Increases in Depressive Symptoms}

Pro-inflammatory IL-1 $\beta$ reactivity was associated with increases in depressive symptoms one year later $(\beta=.621, t(29)=4.261, p<.01)$, and remained significant when controlling for age, BMI, caregiver status, AD use ${ }^{\S}$, basal IL- $1 \beta$ and baseline IDS scores (all $p$ 's <.01; see Table 2 for unadjusted, basal and full models). Entering covariates sequentially to retain power, BMI was the only covariate that was significantly associated with $\Delta \mathrm{IDS}$ ( $\beta=.290$, $t(28)=2.095, p=.045)$. As expected, basal IDS scores were significantly associated with $\Delta \mathrm{IDS}(p<.01)$, such that individuals with higher levels of depressive symptoms at baseline exhibited lesser increases, which may represent a natural course of remission or regression to the mean. No significant caregiver status by IL- $1 \beta$ reactivity interaction was found $(p=$. 549), indicating that this relationship was not moderated by caregiving. Depressive symptoms at the baseline visit were not cross-sectionally associated with circulating levels of IL-1 $\beta(p=.601)$.

\footnotetext{
\$Changes in $\mathrm{AD}$ use were significantly associated with changes in depression in the expected direction $(\mathrm{n}=5, p=.010)$. However, statistically controlling for changes in AD use or dropping participants taking ADs did not affect the pattern of significance linking POS and $\triangle \mathrm{IDS}$.

$\S_{\text {IL-1 }} \beta$ remained a significant predictor of $\Delta$ IDS when either 1) statistically controlling for basal AD use and changes in AD use or 2) dropping participants taking ADs from the analysis.
} 


\section{Mediation Analyses}

We additionally tested the possibility that pro-inflammatory reactivity mediated the relationship between maintenance of a positive outlook during stress and future increases in depressive symptoms. No covariates were included, as they did not affect the significance of the main results and would reduce power. The significance of the indirect mediation path was tested using a Sobel Test (1982), which was bootstrapped in order to remedy any concerns about the power of mediation tests in small samples or assumptions about the sampling distribution of the indirect effect (Hayes, 2009). The indirect effect was significant (Indirect path coefficient $B(S E)=-1.194(0.549), p=.030^{* *}$; Figure 2.)

\section{Tests of Cytokine Specificity}

We hypothesized that IL-1 $\beta$ is a particularly important cytokine for regulating neuroinflammatory and behavioral responses to stress. To explore the specificity of IL-1 $1 \beta$, we also examined whether IL-6 reactivity was associated with POS decline and found no significant association. In addition, IL-6 reactivity was not a significant predictor of $\Delta$ IDS, alone, or in the full model (full model: $\beta=.254, t(25)=1.539, p=.137$ ), although the direction of the effect was similar. While it is possible that IL-6 may have been a significant predictor of $\Delta$ IDS with a greater sample size, IL- $1 \beta$ had a larger effect in this sample.

\section{Discussion}

These data are the first to demonstrate that individual differences in the maintenance of a positive outlook in the midst of a stressful experience are associated with stress-induced proinflammatory IL- $1 \beta$ reactivity, which in turn, prospectively predicts future increases in depressive symptoms one year later among post-menopausal women. These results contribute to and extend the growing body of evidence that suggests the possibility that proinflammatory cytokines may play a causal role in depression (Dantzer et al., 2008; Miller et al., 2009). While preliminary, these data raise the possibility that individuals who maintain hope, challenge and confidence during stress may be at lower risk for future depressive symptoms. If confirmed and further developed, this line of research could lead to novel integrative treatments, potentially targeting mastery or stress-resilience on one hand and inflammatory responses on the other.

Including the current study, three separate studies of older adults have now found that proinflammatory cytokines of the IL-1 family were prospectively associated with the development of or increases in future depressive symptoms (Milaneschi et al., 2009; van den Biggelaar et al., 2007), although the methods of cytokine assessment varied (circulating, in vitro, or acute stress-induced reactivity). In a previous study of older adults, in vitro LPSinduced production of IL-1ra and IL-1 $\beta$ prospectively predicted future increases in depressive symptoms over five years (van den Biggelaar et al., 2007). Another crosssectional study reported higher circulating levels of IL- $1 \beta$ among older adults with MDD compared to controls, which correlated with the duration and severity of the episode

\footnotetext{
**A A trend toward significance was also evident when all covariates were entered ( $p=.09)$, suggesting that including all covariates reduced power but did not drastically change the pattern of results.
} 
(Thomas et al., 2005). Interestingly, the same study found no differences in circulating IL-1 $\beta$ when comparing patients with subsyndromal depression to controls. We also did not find a concurrent relationship between circulating IL- $1 \beta$ levels and depressive symptoms at the baseline visit, which could potentially reflect fact that depressive symptoms were generally subclinical in this sample. In sum, future research should examine the extent to which IL- $1 \beta$ and IL-1ra production in response to either acute laboratory stress or mitogen stimulation may represent a trait-like predisposing factor for depressive disorders.

These findings extend the literature showing that positive affect and cognitions may represent unique components of psychobiological resilience (Marsland et al., 2007; Steptoe et al., 2009). If confirmed, the current data may have implications for the prevention and psychological treatment of depression. Whereas traditional Cognitive Behavioral Therapy for mood disorders focuses on reframing "maladaptive cognitions" that promote negative affect, these data suggest that interventions should additionally target the ability to maintain positive affect during stress. A meta-analysis of 51 positive psychology interventions also concluded that treatment methods that cultivated positive feelings, behaviors and cognitions were effective in decreasing depressive symptoms (Sin and Lyubomirsky, 2009). Resilience is conceptualized as a dynamic process that occurs through interactions with stressors in the environment (Luthar et al., 2000). The validation check comparing this positive outlook measure with the PANAS positive affect scale revealed that the dynamics of a positive outlook during acute stress appear to provide a different index than an average level of positive affect over a week. Thus, assessing changes in positive affect and cognitions during acute stress may provide a snap-shot of psychobiological stress-resilience.

An important contribution of the current study is to help place this PsychoNeurolmmunological model of brain-immune interactions into a clinical and translational context (Irwin and Miller, 2007). Although many studies of acute stressinduced cytokine reactivity have been conducted, only a handful of studies have shown that it predicts important clinical or health outcomes (e.g., Brydon and Steptoe, 2005). The data herein are consistent with a theoretical model in which psychological factors influence cytokine reactivity, which in turn acts on the brain, forming a bidirectional circuit in which brain-immune interactions are quantified with dynamic markers (e.g., stress-induced change) (Aschbacher and Kemeny, 2011).

The present study has several important limitations. First, the current data are based on depressive symptoms, which are subclinical in the sample as a whole, rather than on clinical diagnoses of MDD. Nonetheless, over one-third of the sample at baseline met criteria for subthreshold depression, which is clinically relevant as it is associated with functional impairment (Karsten et al., 2010). A few individuals in the sample were taking antidepressant medications; however, the pattern of results remained significant when AD use was statistically controlled for and also when these individuals were dropped. While no moderating effects of caregiving were found in these analyses, their presence in the sample raises possibility that the results may be influenced by the higher prevalence of chronically stressed individuals. Although the mediation model implies that psychological responses to acute stress influence cytokine reactivity, the true causal direction cannot be inferred from this data and may even be bidirectional. Finally, it is important to recognize that there are 
limitations with multiplex cytokine assays including the one used in this study (Breen et al., 2011), which could potentially explain the failure to detect IL-6 effects. While these findings need further replication and investigation in a larger sample addressing the aforementioned limitations, they are nonetheless a valuable complement to and extension of the existing literature on cytokines and depressive symptoms.

In sum, maintaining a positive outlook during a stressful event may help protect against heightened pro-inflammatory activity and depressive symptoms. To our knowledge, this study is the first to show that individual differences in IL- $1 \beta$ reactivity to acute stress were prospectively associated with increases in depressive symptoms one year later. If replicated, these data suggest that IL- $1 \beta$ reactivity to acute stress may serve as a marker to identify which individuals are at greatest risk for stress-induced depression. Novel integrative approaches to treating depressive symptoms could potentially involve bolstering skills to maintain positivity under stress on the one hand, and pharmacotherapeutic interventions targeting immune responses on the other (Miller et al., 2009; Wolkowitz et al., 2011).

\section{References}

Altemus M, Rao B, Dhabhar FS, Ding W, Granstein RD. Stress-induced changes in skin barrier function in healthy women. J Invest Dermatol. 2001; 117:309-317. [PubMed: 11511309]

Aschbacher K, Kemeny ME. New directions in linking the dynamics of affective and stress-arousal systems. Brain Behav Immun. 2011; 25:230-231. [PubMed: 21044679]

Barth J, Schumacher M, Herrmann-Lingen C. Depression as a risk factor for mortality in patients with coronary heart disease: A meta-analysis. Psychosom Med. 2004; 66:802-813. [PubMed: 15564343]

Bluthe RM, Dantzer R, Kelley KW. Effects of interleukin-1 receptor antagonist on the behavioral effects of lipopolysaccharide in rat. Brain Res. 1992; 573:318-320. [PubMed: 1387028]

Breen EC, Reynolds SM, Cox C, Jacobson LP, Magpantay L, Mulder CB, Dibben O, Margolick JB, Bream JH, Sambrano E, Martinez-Maza O, Sinclair E, Borrow P, Landay AL, Rinaldo CR, Norris PJ. Mutisite comparison of high-sensitivity multiplex cytokine assays. Clin Vaccine Immunol. 2011; 18:1229-1242. [PubMed: 21697338]

Brydon L, Steptoe A. Stress-induced increases in interleukin- 6 and fibrinogen predict ambulatory blood pressure at 3-year follow-up. J Hypertens. 2005; 23:1001-1007. [PubMed: 15834286]

Carroll JE, Low CA, Prather AA, Cohen S, Fury JM, Ross DC, Marsland AL. Negative affective responses to a speech task predict changes in interleukin (IL)-6. Brain Behav Immun. 2011; 25:232238. [PubMed: 20888901]

Cohen S, Hamrick N. Stable individual differences in physiological response to stressors: implications for stress-elicited changes in immune related health. Brain Behav Immun. 2003; 17:407-414. [PubMed: 14583231]

Cuijpers P, Smit F, Oostenbrink J, de Graaf R, ten Have M, Beekman A. Economic costs of minor depression: a population-based study. Acta Psychiat Scand. 2007; 115:229-236. [PubMed: 17302623]

Cuijpers P, Smit H. Excess mortality in depression: a meta-analysis of community studies. J Affect Disorders. 2002; 72:227-236. [PubMed: 12450639]

Dantzer R, O'Connor JC, Freund GG, Johnson RW, Kelley KW. From inflammation to sickness and depression: when the immune system subjugates the brain. Nat Rev Neurosci. 2008; 9:46-57. [PubMed: 18073775]

DellaGioia N, Hannestad J. A critical review of human endotoxin administration as an experimental paradigm of depression. Neurosci Biobehav R. 2010; 34:130-143.

Dhabhar FS, Burke HM, Epel ES, Mellon SH, Rosser R, Reus VI, Wolkowitz OM. Low serum IL-10 concentrations and loss of regulatory association between IL-6 and IL-10 in adults with major depression. J Psychiatr Res. 2009; 43:962-969. [PubMed: 19552919] 
Dinarello CA. Immunological and Inflammatory Functions of the Interleukin-1 Family. Annu Rev Immunol. 2009; 27:519-550. [PubMed: 19302047]

Epel E, Daubenmier J, Moskowitz JT, Folkman S, Blackburn E. Can Meditation Slow Rate of Cellular Aging? Cognitive Stress, Mindfulness, and Telomeres. Longevity, Regeneration, and Optimal Health: Integrating Eastern and Westen Perspectives. 2009; 1172:34-53.

Epel ES, McEwen BS, Ickovics JR. Embodying psychological thriving: Physical thriving in response to stress. J Soc Issues. 1998; 54:301-322.

Goshen I, Kreisel T, Ben-Menachem-Zidon O, Licht T, Weidenfeld J, Ben-Hur T, Yirmiya R. Brain interleukin-1 mediates chronic stress-induced depression in mice via adrenocortical activation and hippocampal neurogenesis suppression. Mol Psychiatry. 2008; 13:717-728. [PubMed: 17700577]

Hayes AF. Beyond Baron and Kenny: Statistical Mediation Analysis in the New Millennium. Commun Monogr. 2009; 76:408-420.

Irwin MR, Miller AH. Depressive disorders an immunity: 20 years of progress and discovery. Brain Behav Immun. 2007; 21:374-383. [PubMed: 17360153]

Karsten J, Hartman CA, Ormel J, Nolen WA, Penninx BW. Subthreshold depression based on functional impairment better defined by symptom severity than by number of DSM-IV symptoms. J Affect Disord. 2010; 123:230-237. [PubMed: 19896201]

Kendler KS, Karkowski LM, Prescott CA. Stressful life events and major depression: risk period, long-term contextual threat, and diagnostic specificity. J Nerv Ment Dis. 1998; 186:661-669. [PubMed: 9824167]

Kirschbaum C, Pirke KM, Hellhammer DH. The 'Trier Social Stress Test'--a tool for investigating psychobiological stress responses in a laboratory setting. Neuropsychobiology. 1993; 28:76-81. [PubMed: 8255414]

Luthar SS, Cicchetti D, Becker B. The construct of resilience: a critical evaluation and guidelines for future work. Child Dev. 2000; 71:543-562. [PubMed: 10953923]

Maes M, Bosmans E, De Jongh R, Kenis G, Vandoolaeghe E, Neels H. Increased serum IL-6 an IL-1 receptor antagonist concentrations in major depression and treatment resistant depression. Cytokine. 1997; 9:853-858. [PubMed: 9367546]

Marsland, AL.; Pressman, S.; Cohen, S., editors. Positive affect and immune function. Academic Press; San Diego, CA: 2007.

Matthews KA, Katholi CR, McCreath H, Whooley MA, Williams DR, Zhu S, Markovitz JH. Blood pressure reactivity to psychological stress predicts hypertension in the CARDIA study. Circulation. 2004; 110:74-78. [PubMed: 15210592]

Mendes WB, Blascovich J, Major B, Seery M. Challenge and threat responses during downward an upward social comparisons. Eur J Soc Psychol. 2001; 31:477-497.

Milaneschi Y, Corsi AM, Penninx BW, Bandinelli S, Guralnik JM, Ferrucci L. Interleukin-1 receptor antagonist and incident depressive symptoms over 6 years in older persons: the InCHIANTI study. Biol Psychiatry. 2009; 65:973-978. [PubMed: 19111279]

Miller AH, Maletic V, Raison CL. Inflammation and its discontents: the role of cytokines in the pathophysiology of major depression. Biol Psychiatry. 2009; 65:732-741. [PubMed: 19150053]

Miller GE, Rohleder N, Stetler C, Kirschbaum C. Clinical depression and regulation of the inflammatory response during acute stress. Psychosom Med. 2005; 67:679-687. [PubMed: 16204423]

Nguyen KT, Deak T, Owens SM, Kohno T, Fleshner M, Watkins LR, Maier SF. Exposure to acute stress induces brain interleukin-1beta protein in the rat. J Neurosci. 1998; 18:2239-2246. [PubMed: 9482808]

Pace TW, Mletzko TC, Alagbe O, Musselman DL, Nemeroff CB, Miller AH, Heim CM. Increased stress-induced inflammatory responses in male patients with major depression and increased early life stress. Am J Psychiatry. 2006; 163:1630-1633. [PubMed: 16946190]

Rush AJ, Giles DE, Schlesser MA, Fulton CL, Weissenburger J, Burns C. The Inventory of Depressive Symptomatology (Ids) - Preliminary Findings. Psychopharmacol Bull. 1986; 22:985-990.

Rush AJ, Gullion CM, Basco MR, Jarrett RB, Trivedi MH. The Inventory of Depressive Symptomatology (IDS): Psychometric properties. Psychol Med. 1996; 26:477-486. [PubMed: 8733206] 
Rush AJ, Trivedi MH, Wisniewski SR, Nierenberg AA, Stewart JW, Warden D, Niederehe G, Thase ME, Lavori PW, Lebowitz BD, McGrath PJ, Rosenbaum JF, Sackeim HA, Kupfer DJ, Luther J, Fava M. Acute and longer-term outcomes in depressed outpatients requiring one or several treatment steps: A STAR*D report. Am J Psychiat. 2006; 163:1905-1917. [PubMed: 17074942]

Sin NL, Lyubomirsky S. Enhancing well-being and alleviating depressive symptoms with positive psychology interventions: a practice-friendly meta-analysis. J Clin Psychol. 2009; 65:467-487. [PubMed: 19301241]

Sobel M. The Conditional Level of Confidence-Intervals for the Normal Variance and Applications. Commun Stat Theory. 1989; 18:2813-2823.

Sobel ME. Asymptotic confidence intervals for indirect effects in structural equations models. Sociol Methodol. 1982; 13:290-312.

Steptoe A, Dockray S, Wardle J. Positive affect and psychobiological processes relevant to health. J Pers. 2009; 77:1747-1776. [PubMed: 19796062]

Steptoe A, Hamer M, Chida Y. The effects of acute psychological stress on circulating inflammatory factors in humans: A review and meta-analysis. Brain Behav Immun. 2007; 21:901-912. [PubMed: 17475444]

Stewart WF, Ricci JA, Chee E, Hahn SR, Morganstein D. Cost of lost productive work time among US workers with depression. JAMA. 2003; 289:3135-3144. [PubMed: 12813119]

Thomas AJ, Davis S, Morris C, Jackson E, Harrison R, O'Brien JT. Increase in interleukin-1beta in late-life depression. Am J Psychiatry. 2005; 162:175-177. [PubMed: 15625217]

Tomaka J, Blascovich J, Kibler J, Ernst JM. Cognitive and physiological antecedents of threat and challenge appraisal. J Pers Soc Psychol. 1997; 73:63-72. [PubMed: 9216079]

Unutzer J, Schoenbaum M, Katon WJ, Fan MY, Pincus HA, Hogan D, Taylor J. Healthcare Costs Associated with Depression in Medically Ill Fee-for-Service Medicare Participants. Journal of the American Geriatrics Society. 2009; 57:506-510. [PubMed: 19175438]

van den Biggelaar AH, Gussekloo J, de Craen AJ, Frolich M, Stek ML, van der Mast RC, Westendorp RG. Inflammation and interleukin-1 signaling network contribute to depressive symptoms but not cognitive decline in old age. Exp Gerontol. 2007; 42:693-701. [PubMed: 17350781]

Watson D, Cark LA, Tellegen A. Development and validation of brief measures of positive and negative affect: the PANAS scales. J Pers Soc Psychol. 1988; 54:1063-1070. [PubMed: 3397865]

Wolkowitz OM, Reus VI, Mellon SH. Of sound mind and body: depression, disease, and accelerated aging. Dialogues Clin Neurosci. 2011; 13:25-39. [PubMed: 21485744] 
$\longrightarrow$ Decreasing POS $\because \varpi \cdot$ Increasing POS

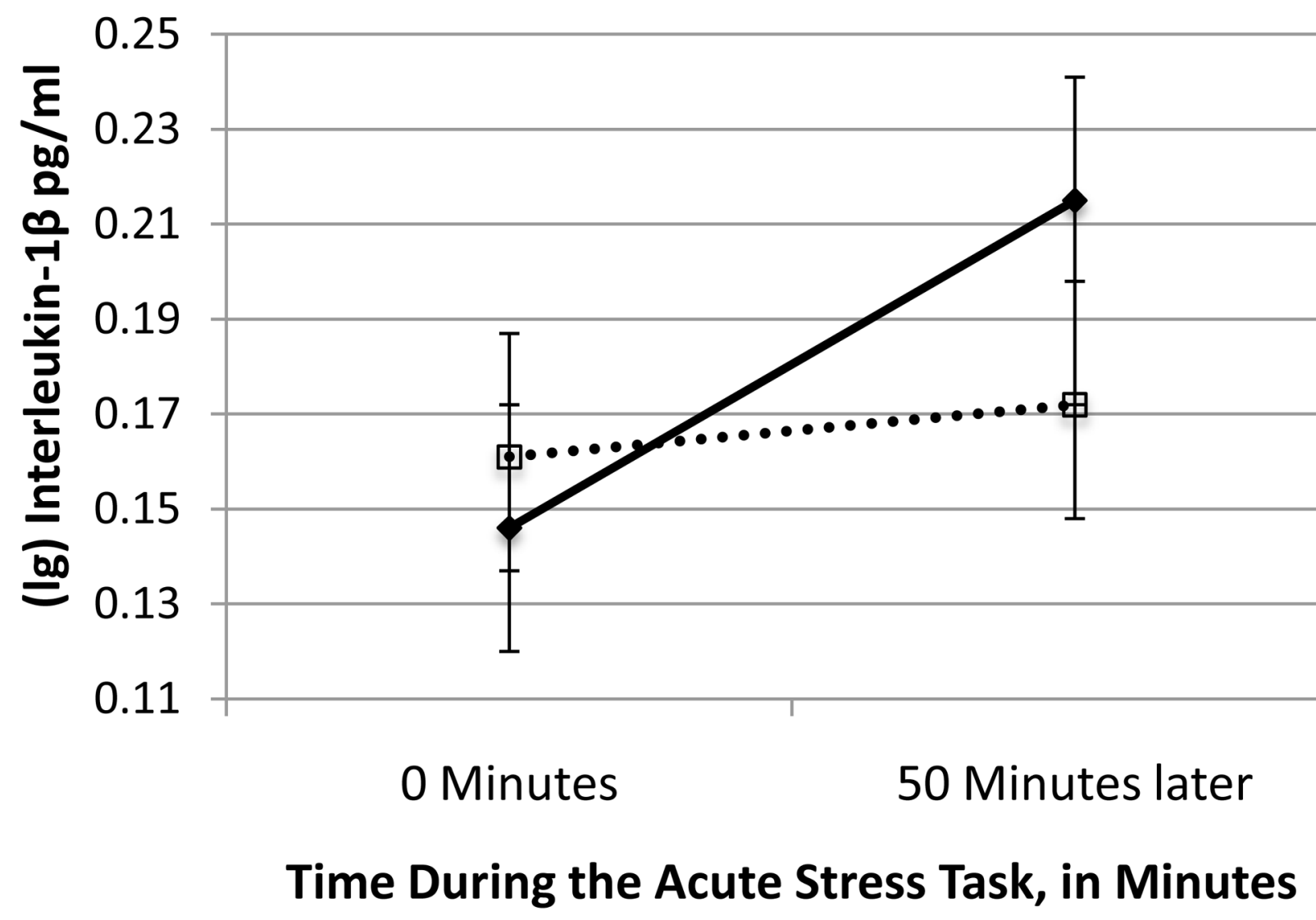

Figure 1. The Decrease in a Positive Outlook During Acute Stress is Associated with Greater Pro-Inflammatory Interleukin-1 $\beta$ Reactivity

POS = Positive Affect and Cognitions. The analyses reported in the results use the continuous measures of acute-stress induced change in POS to predict 50-minute IL-1 $\beta$ reactivity $(p=.017)$. In this figure, the effects are represented dichotomously to better illustrate how individuals $(n=19)$ whose POS decreased in resonse to acute stress had a greater increase in IL-1 $\beta$, relative to individuals $(n=16)$ whose POS increased or stayed the same following the acute stress task. 


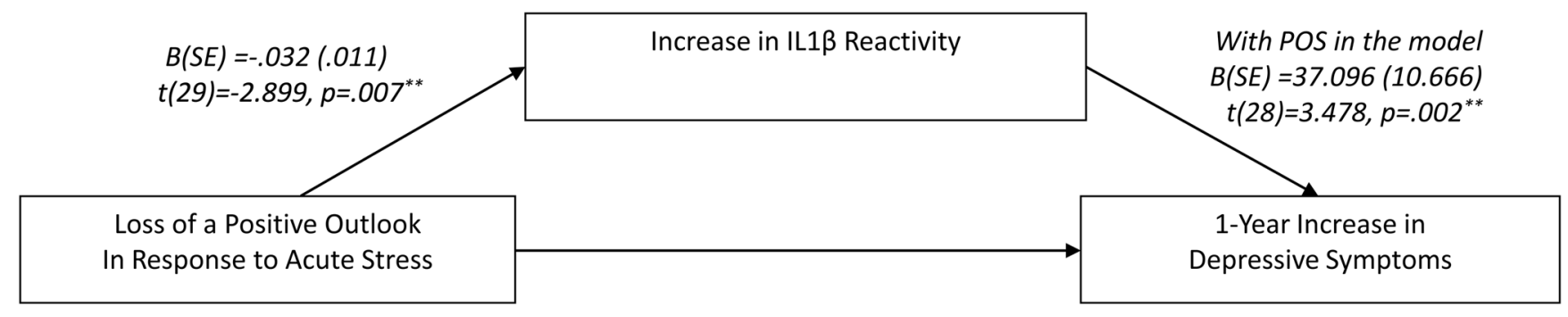

$B(S E)=-1.535(.750)$,

$t(29)=-2.047, p=.050^{*}$

Figure 2. Pro-inflammatory IL1 $\beta$ Reactivity Mediates the Relationship between Loss of Positivity During Acute Stress \& Future Depressive Symptom Increases

$* * p \leq 01, * p \leq 05$. Bootstrapped linear regression analyses of the indirect effect (Hayes,

2009; Sobel, 1989) were used to test the hypothesis that pro-inflammatory reactivity to acute stress is a significant mediator of the relationship between loss of a positive outlook during acute stress and prospective risk of depressive symptom increases. Loss of a positive outlook was quantified as the decrease in Positive Affect and Cognitions (POS) during the acute stress task. The indirect effect was significant: $B(S E)=-1.194(0.549), p=.030 *$. 


\section{Table 1}

Absolute Cytokine Levels before and after the TSST

\begin{tabular}{|l|c|c|}
\hline$(\mathbf{N}=\mathbf{3 5})$ & Interleukin-1 $\boldsymbol{\beta}, \mathbf{p g} / \mathbf{m l}$ & Interleukin-6, pg/ml \\
\hline${\text { TSST baseline, } \mathrm{M}(\mathrm{SD})^{*}}^{*}$ & $0.17(0.14)$ & $4.01(13.77)$ \\
\hline 50 minutes after TSST baseline, M(SD) & $0.23(0.24)$ & $5.13(14.74)$ \\
\hline
\end{tabular}

* Note: The means and their standard deviations (M(SD)), given here, are intended merely to provide a rough benchmark of overall levels, but should not be taken as a statistical estimate of central tendency due to the skewed nature of cytokine data prior to log-transformation. All other analyses presented herein utilize log-transformed variables. TSST $=$ Trier Social Stress Task. 
Table 2

Pro-inflammatory Reactivity to Acute Stress at the Baseline Visit Predicts Increases in Depressive Symptoms One Year Later

\begin{tabular}{|l|l|l|l|}
\hline Outcome: One-Year Increase in Depressive Symptoms & Standardized Coefficient $(\boldsymbol{\beta})$ & T-test $(\mathbf{d f})$ & P-value \\
\hline Unadjusted Model & & $(\mathrm{Df}=29)$ & \\
\hline Interleukin (IL)-1 $\beta$ Reactivity & 0.621 & 4.261 & $0.001^{* *}$ \\
\hline Model Adjusting for Basal Factors & & $(\mathrm{Df}=22)$ & \\
\hline Fasting Basal IL1B & -0.068 & -0.490 & 0.629 \\
\hline Basal Depressive Symptoms & -0.361 & -2.602 & $0.016^{*}$ \\
\hline IL-1 $\beta$ Reactivity & 0.609 & 4.347 & $0.001^{* *}$ \\
\hline Full Model Adjusting for Covariates & & $(\mathrm{Df}=25)$ & \\
\hline Age & 0.068 & 0.468 & 0.644 \\
\hline Body Mass Index & 0.284 & 1.951 & $0.062^{\dagger}$ \\
\hline Caregiving & 0.216 & 1.255 & 0.221 \\
\hline Antidepressant Use & 0.005 & 0.034 & 0.973 \\
\hline IL-1 $\beta$ Reactivity & 0.501 & 3.227 & $0.003^{*}$ \\
\hline
\end{tabular}

**

$p \leq 01$,

*

* $p \leq 05$

${ }^{\dagger} p \leq 07$, critical alpha $=.05$.

$\mathrm{Df}=$ degrees of freedom. All models were inputted as regression analyses, in which covariates or basal factors were entered in block 1 and IL-1 $\beta$ reactivity was entered in block 2 . IL- $1 \beta$ Reactivity represents the change in IL-1 $\beta$ levels from right before the acute stress task to 50 minutes later. Increases in depressive symptoms were quantified as the increase in symptom scores on the Inventory for Depressive Symptoms from the baseline visit to one year later. 